\title{
Research Article \\ Size-Selective Precipitation and Aggregate Reduction of FePt-Based Nanoparticles
}

\author{
Pharunee Sarmphim, ${ }^{1}$ Pongsakorn Jantaratana, ${ }^{2}$ and Chitnarong Sirisathitkul ${ }^{1}{ }^{1}$ \\ ${ }^{1}$ Center of Excellence in Functional Materials and Nanotechnology, School of Science, Walailak University, \\ Nakhon Si Thammarat 80161, Thailand \\ ${ }^{2}$ Department of Physics, Faculty of Science, Kasetsart University, Bangkok 10900, Thailand
}

Correspondence should be addressed to Chitnarong Sirisathitkul; chitnarong.siri@gmail.com

Received 15 April 2018; Accepted 27 June 2018; Published 17 July 2018

Academic Editor: Paulo Cesar Morais

Copyright (c) 2018 Pharunee Sarmphim et al. This is an open access article distributed under the Creative Commons Attribution License, which permits unrestricted use, distribution, and reproduction in any medium, provided the original work is properly cited.

\begin{abstract}
Magnetic nanoparticles with a narrow size distribution are desirable for applications in ultra-high density data storage and biomedicine. In this work, the size-selective precipitation and aggregate reduction were combined to classify superparamagnetic iron platinum- (FePt-) based nanoparticles. The size-selective precipitation was implemented with the variation in the amount of ethanol. In the first condition, the ratio of ethanol-to-nanosuspension of $3: 4$ was used in the precipitation twice. By contrast, the second condition employed the ratios of ethanol-to-nanosuspension of $4: 4$ in the first precipitation and $5: 4$ in the second precipitation. The first precipitation successfully sorted out the aggregated particles, and the second precipitation collected particles with a narrow size distribution. The increase in ethanol enhanced the monodispersity of nanoparticles as shown by transmission electron microscopy (TEM) images and size distribution curves. In addition, large aggregated by-products from the precipitation stage were sonicated with the addition of surfactants. The increase in ultrasonic power reduces the aggregation, but the longer sonication led to an uneven distribution.
\end{abstract}

\section{Introduction}

Colloidal magnetic nanoparticles stabilized in carrier liquids, referred to as nanosuspension, have been implemented in engineering and biomedicine. Most applications require a narrow size distribution and a dispersion without agglomeration. In addition, a good liquid conformation usually leads to a long-range order on the substrate conformation in applications such as sensors and data storage. However, the chemical synthesis often results in polydisperse nanoparticles. Moreover, the aggregation arising from particles rigidly joined together in a suspension destabilizes the nanosuspension. Therefore, surfactants are added to control the size and dispersion of nanoparticles. For surface-modified magnetic nanoparticles, the stability depends upon the balance of the attractive force (magnetic dipolar and van der Waals interactions) and the repulsive force (electrostatic and electrosteric interactions) [1]. Nonuniform composition and shape of magnetic nanoparticle lead to aggregates due to the imbalance of dipolar attraction.

After the synthesis and purification, the monodispersity of nanoparticles dispersed in carrier liquids can be improved by the separation techniques. A variety of separation techniques include magnetic separation, chromatography, density gradient centrifugation, electrophoresis, and size-selective precipitation [2]. The size-selective precipitation is a facile technique to separate polydisperse nanoparticles into fractions of narrower size distributions [3]. After that, large aggregated particles can be separated from the smaller particles during the centrifugation. Polar antisolvents such as ethanol and methanol have been employed in the precipitation $[4,5]$. However, the use of the antisolvent can substantially remove surfactants from nanoparticles [6]. In addition to surface modification of ferrite nanoparticles, $\mathrm{Li}$ et al. [7] used oleic acid to yield the nanoparticle size distribution with the standard derivation of less than $9 \%$ from the 
size-selective precipitation. In another work, the saltinduced precipitation was proven effective in classifying gold nanoparticles [8].

The large aggregated by-products removed from the size-selective precipitation can be exploited in repeated size-selective precipitation as well as aggregate reduction. Ultrasound-assisted approaches can break the aggregated particles by shock waves and induced cavitation $[9,10]$. Furthermore, the sonication with a high shear force disperses surface-modified nanoparticles in carrier liquids [11].

This work combines the size-selective precipitation and aggregate reduction to classify iron platinum- (FePt-) based nanoparticles. The green synthesis using alternative precursors to $\mathrm{Fe}(\mathrm{CO})_{5}$ often gives rise to products with a wide size distribution [12]. Polydisperse nanoparticles firstly underwent the size-selective precipitation. The fraction with a narrower size distribution was selected whereas larger aggregates were further reduced by means of ultrasonic irradiation.

\section{Experimental}

2.1. Synthesis of Nanoparticles. Nanoparticles were prepared from the reaction between $0.1 \mathrm{mmol}$ platinum(II) acetylacetonate $\left(\mathrm{Pt}(\mathrm{acac})_{2}\right)$ and $0.12 \mathrm{mmol}$ iron(III) tris (2,2,6,6-tetramethyl-3,5-heptanedionate) (Fe (tmhd) $)_{3}$ ) in a Schlenk flask. These precursors were mixed with $10 \mathrm{~mL}$ dioctyl ether under nitrogen atmosphere. The obtained mixture was then heated to $120^{\circ} \mathrm{C}$ and dwelled at this temperature for $20 \mathrm{~min}$. After the addition of $2.5 \mathrm{~mL}$ oleic acid and $0.2 \mathrm{~mL}$ oleylamine surfactants, the mixture was further heated at $200^{\circ} \mathrm{C}$ for $30 \mathrm{~min}$ to assure that the precursors were completely decomposed. The mixture was allowed to reflux at $296^{\circ} \mathrm{C}$ for $30 \mathrm{~min}$ and then cooled down to room temperature. The obtained nanoparticles were repeatedly separated and purified by the centrifugation and then dispersed in n-hexane to obtain a concentration around $2 \mathrm{mg} / \mathrm{mL}$. The elemental composition and magnetic properties of as-synthesized nanoparticles were, respectively, measured by energy dispersive spectroscopy (EDS) and vibrating sample magnetometry (VSM).

2.2. Size-Selective Precipitation. Half $\mathrm{mL}$ of ethanol, $6 \mu \mathrm{L}$ of oleic acid, and $6 \mu \mathrm{L}$ of oleylamine were added in $0.5 \mathrm{~mL}$ of $a s$-synthesized sample. Then, the mixtures were centrifuged at $5000 \mathrm{rpm}$ for $15 \mathrm{~min}$ leading to precipitates. The precipitates were dried, weighted, and finally suspended in hexane to obtain a nanosuspension of $0.2 \mathrm{mg} / \mathrm{mL}$, referred to as sample A and sample B.

The size-selective precipitation was carried out by adjusting the amount of ethanol into sample A1 and sample A2. For sample A1, $3 \mathrm{~mL}$ of ethanol was added to $4 \mathrm{~mL}$ of sample $\mathrm{A}$, hence the volume ratio of $3: 4$. Then, the solution was centrifuged to separate precipitates from supernatant. The precipitates were dried and weighted. The solid yield of $0.3 \mathrm{mg}$ was dispersed in $1.5 \mathrm{~mL}$ hexane to obtain a nanosuspension with the addition of oleic acid and oleylamine, referred to as sample A1. To prepare sample A2, the volume ratio of $3: 4$ was obtained by adding $3 \mathrm{~mL}$ of ethanol to the nanosuspension. After the centrifugation and the removal of supernatant, the drying and dispersion were repeated. The solid

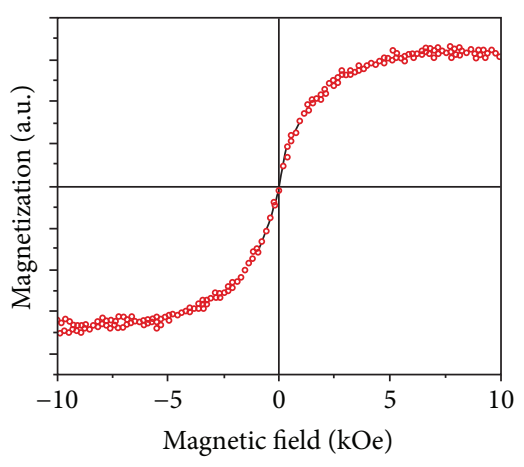

FIGURE 1: Magnetization curve of as-synthesized particles measured by VSM.

yield of $0.4 \mathrm{mg}$ was redispersed in $2 \mathrm{~mL}$ hexane to obtain a nanosuspension with the addition of oleic acid and oleylamine, referred to as sample A2.

To investigate the variation in ethanol, sample B1 and sample B2 were obtained from the first and second precipitations, respectively, with different conditions. Four $\mathrm{mL}$ of ethanol was added to $4 \mathrm{~mL}$ of sample $\mathrm{B}$ in the first centrifugation for sample B1, and sample B2 was obtained by using the volume of ethanol to the nanosuspension of $5: 4$.

2.3. Aggregate Reduction. Sample A1 and sample B1 with remaining aggregates were combined into sample $\mathrm{C}$ for the aggregate reduction process. Two $\mathrm{mL}$ of sample $\mathrm{C}$ (concentration of $0.2 \mathrm{mg} / \mathrm{mL}$ ) was sonicated at $42-45 \mathrm{kHz}$ using the maximum electrical power around $200 \mathrm{~W}$ (Crest CP1200 Ultrasonic). During the sonication, $10 \mu \mathrm{L}$ of oleic acid and oleylamine were added and the sonication was carried out for $30 \mathrm{~min}$. To determine the process parameters, the experiment was repeated without the addition of oleic acid and oleylamine. The power level of $170 \mathrm{~W}$ was also tested, and the sonicated time of $60 \mathrm{~min}$ was compared to $30 \mathrm{~min}$.

Each sample was dropped on a grid substrate and inspected by transmission electron microscopy (TEM; JEOL, JEM-2010) at an accelerating voltage of $200 \mathrm{kV}$. TEM images were then analyzed by ImageJ program to determine the particle size distribution.

\section{Results and Discussion}

3.1. Properties of as-Synthesized Nanoparticles. The as-synthesized particles are stabilized by the tendency of Fe-oleic acid and Pt-oleylamine binding [13]. The dispersion in nonpolar hydrophobic solvents such as hexane is a commonplace for nanoparticles with these organic ligands [14], but the water-based nanosuspension is advantageous in biomedical applications. As suggested in [15], surface modification of FePt by aminoethanethiol can be achieved with water solubility via a ligand exchange process.

The EDS spectrum, not shown here, exhibits $\mathrm{C}$ and $\mathrm{O}$ peaks attributed to the TEM grid substrate and surfactants in addition to Fe and Pt detections. The composition, averaged from 3 positions, indicates a much higher fraction of Pt $(\sim 60 \%)$ over $\mathrm{Fe}(\sim 15 \%)$. Magnetic properties of dried nanoparticles are represented by Figure 1 . The variation in 


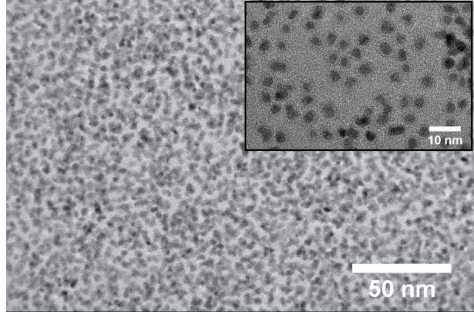

(a)

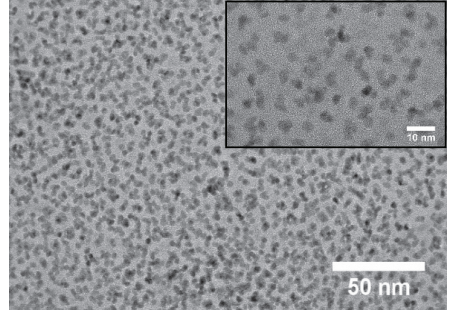

(b)

FIGURE 2: TEM image of as-synthesized particles from the nanosuspension with (a) high concentration of $2 \mathrm{mg} / \mathrm{mL}$ and (b) low concentration of $0.2 \mathrm{mg} / \mathrm{mL}$. Insets are magnified images.

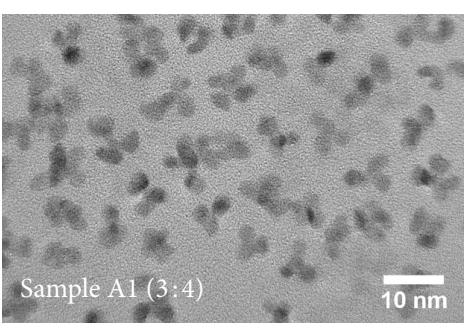

(a)

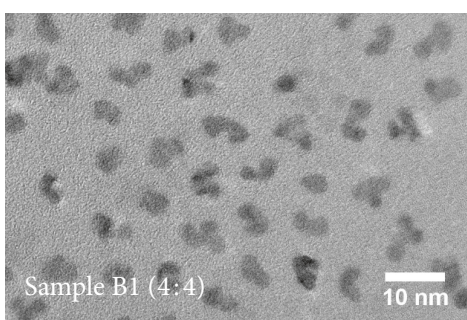

(b)

FIGURE 3: TEM images of large precipitates from the first precipitation using ethanol: nanosuspension ratio of (a) $3: 4$ (sample A1) and (b) $4: 4$ (sample B1).

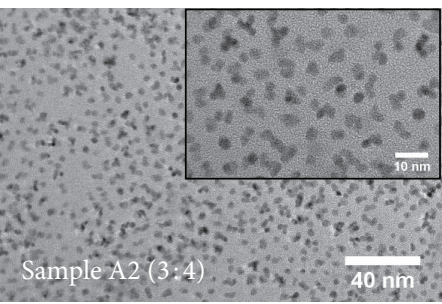

(a)

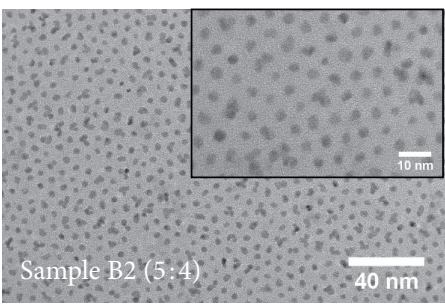

(b)

FIGURE 4: TEM images of particles from the second precipitation using ethanol: nanosuspension ratio of (a) $3: 4$ (sample A2) and (b) $5: 4$ (sample B2).

magnetization in changing fields up to $10 \mathrm{kOe}$ without hysteresis is a superparamagnetic characteristic.

3.2. Size-Selective Precipitation. Before the size-selective precipitation, most as-synthesized particles in Figure 2(a) are overlapped due to the magnetic interaction in concentrated nanosuspension $(2 \mathrm{mg} / \mathrm{mL})$. Only some areas show the separation of particles as shown in the highly magnified inset of Figure 2(a). The nanoparticles can be approximated as spheres with diameter around $5 \mathrm{~nm}$. In Figure 2(b), the average spacing between nanoparticles is increased by the dilution of the nanosuspension to $0.2 \mathrm{mg} / \mathrm{mL}$. However, the overlapping particles are not separated, and the sizeselective precipitation is therefore necessary. After the size-selective precipitation, samples $\mathrm{A} 1$ and $\mathrm{B} 1$ from the first precipitation are compared in Figure 3. Both samples contain aggregates larger than $5 \mathrm{~nm}$ in diameter, but the larger amount of ethanol (Sample B1) leads to more isolated nanoparticles.
The amount of ethanol shows a pronounced effect in the second precipitation as shown in Figure 4. Nanoparticles of smaller size can be uniformly distributed in Figure 4(b) by using larger amount of ethanol in the size-selective precipitation. The ethanol:nanosuspension ratio of $4: 4$ in the first precipitation allows the separation of aggregation, and the ratio of $5: 4$ in the second precipitation collects smaller particles with uniform size distribution. The size distribution in forms of log-normal curves and the mean diameters with standard deviation of as-synthesized nanoparticles are compared to those from the second precipitation in Figure 5. Whereas the as-synthesized nanoparticles have a broad distribution up to $8 \mathrm{~nm}$, nanoparticles from the size-selective precipitation exhibit narrower curves with negligible particles beyond $7 \mathrm{~nm}$. By using a larger amount of ethanol, the average diameter of sample B2 is reduced to $3.56 \mathrm{~nm}$ and about half of detected nanoparticles have a diameter ranging between 3 and $4 \mathrm{~nm}$. The effect of ethanol is consistent with other nanoparticles in the literature [16]. 


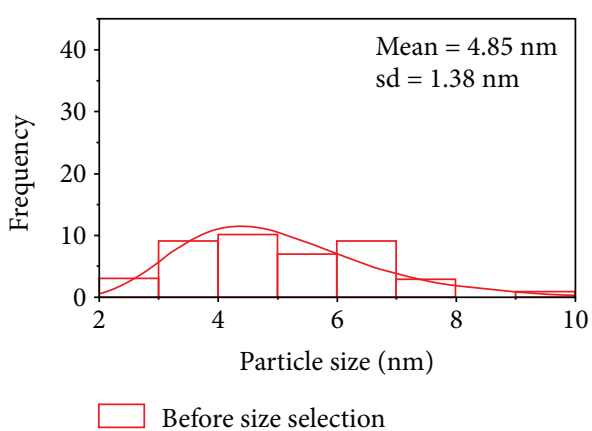

(a)

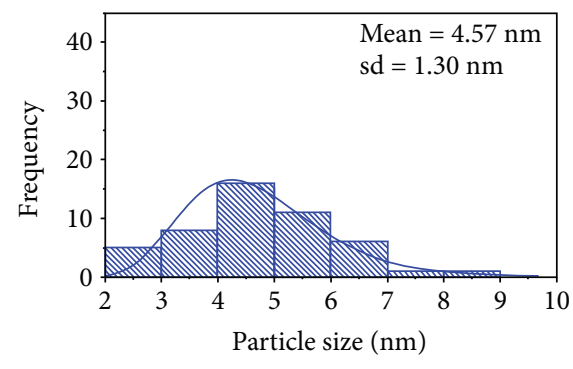

After size selection first condition

(b)

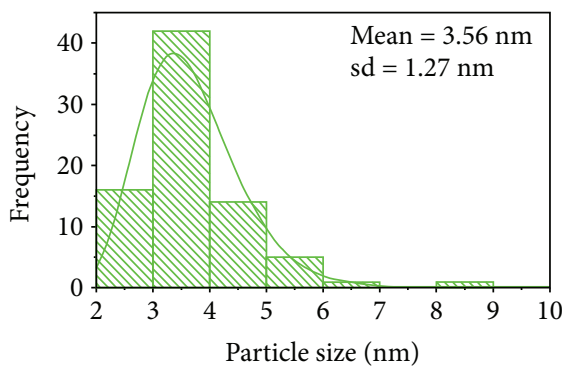

After size selection second condition

(c)

FIGURE 5: Size distribution of FePt nanoparticles: (a) before the size-selective precipitation, (b) sample A2 from the second precipitation using smaller amount of ethanol (the first condition), and (c) sample B2 from the second precipitation using higher amount of ethanol (the second condition).

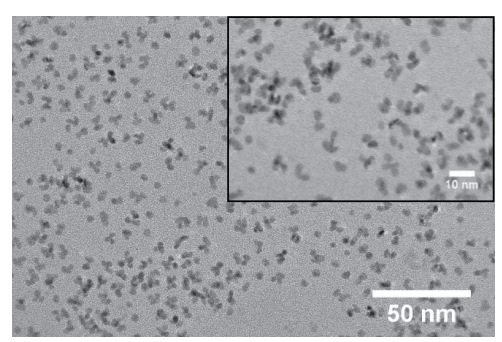

(a)

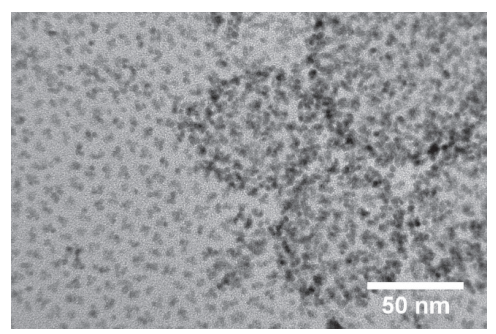

(c)

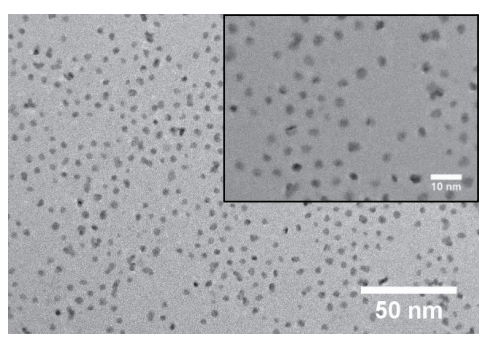

(b)

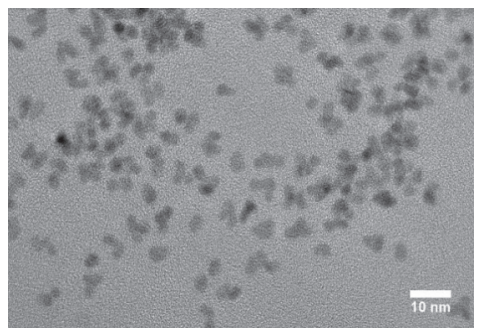

(d)

FIgURE 6: TEM image of sample $\mathrm{C}$ after sonicating at $200 \mathrm{~W}$ (a) without surfactants and (b) with addition of oleic acid and oleylamine for 30 min (insets are magnified images). The effect of sonication time and power is compared with TEM images in (c) with a longer time of $60 \mathrm{~min}$ and (d) with a smaller power of $170 \mathrm{~W}$.

3.3. Aggregate Reduction. Sample C, a combination of larger particles from sample A1 and sample B1, was sonicated in order to reduce the agglomeration. At the same sonication power and time (200 W and $30 \mathrm{~min}$ ), overlapping particles still exist in Figure 6(a) after sonication without surfactants whereas the additions of oleic acid and oleylamine result in less aggregation and no overlapping particles in Figure 6(b). This marked difference clearly suggests that the surfactants facilitate the aggregate reduction. However, a prolonged sonication can have adverse effect on the aggregate reduction. In 
Figure 6(c), the sonication for $60 \mathrm{~min}$ leads to the formation of aggregated clusters and uneven distribution. The agglomeration is likely due to the collision by energy supplied and removal of surfactants [9-11]. Finally, the influence of sonication power on the aggregation is shown in Figure 6(d). With the same surfactant addition, more aggregates remain in sample $\mathrm{C}$ after the $30 \mathrm{~min}$ sonication at a lower power of $170 \mathrm{~W}$.

By using both size-selective precipitation and aggregation reduction, a substantial yield of nanoparticles compared to precursors are obtained. The nanoparticles with narrower size distribution is of order of $\mathrm{mg}$ in this work, but the production scale can be enhanced. The increase in precursors and the size-selective precipitation are straightforward; however, the effect of sonication on aggregate reduction by sonication over much larger volume of nanosuspension needs further tests.

\section{Conclusion}

The size-selective precipitation was used to improve the monodispersity of FePt-based nanoparticles synthesized from the reaction of $\mathrm{Fe}$ (tmhd) $)_{3}$ and $\mathrm{Pt}(\mathrm{acac})_{2}$. TEM was used to visualize the size and morphology of the nanoparticles, and log-normal size distribution curves were obtained from the analysis of TEM images by ImageJ program. The increased ethanol in the first precipitation enabled more separation of large aggregates, and the further increase in the ratio of ethanol-to-nanosuspension in the second precipitation promoted the collection of smaller particles with narrow size distribution around $3.5 \mathrm{~nm}$. Furthermore, the size of aggregates from the first precipitation was reduced by the sonication with addition of surfactants. Effects of sonication power and time were also demonstrated.

\section{Data Availability}

The data used to support the findings of this study are available from the corresponding author upon request.

\section{Conflicts of Interest}

The authors have no potential conflicts of interest.

\section{Acknowledgments}

This work is financially supported by Thailand Excellent Center in Physics (ThEP-60-PIP-WU3). The authors would like to thank K. Chokprasombat of Thaksin University and P. Harding of Walailak University for their suggestions. The technical assistance in TEM imaging by P. Pinsrithong of Scientific Equipment Center, Prince of Songkla University, is acknowledged.

\section{References}

[1] S. Ghosh, W. Jiang, J. D. McClements, and B. Xing, "Colloidal stability of magnetic iron oxide nanoparticles: influence of natural organic matter and synthetic polyelectrolytes," Langmuir, vol. 27, no. 13, pp. 8036-8043, 2011.
[2] B. Kowalczyk, I. Lagzi, and B. A. Grzybowski, "Nanoseparations: strategies for size and/or shape-selective purification of nanoparticles," Current Opinion in Colloid \& Interface Science, vol. 16, no. 2, pp. 135-148, 2011.

[3] S. A. Tovstun and V. F. Razumov, "Theory of size-selective precipitation," Journal of Nanoparticle Research, vol. 19, no. 1, p. 8, 2017.

[4] S. Sun, C. B. Murray, D. Weller, L. Folks, and A. Moser, "Monodisperse FePt nanoparticles and ferromagnetic FePt nanocrystal superlattices," Science, vol. 287, no. 5460, pp. 1989-1992, 2000.

[5] T. Hyeon, "Chemical synthesis of magnetic nanoparticles," Chemical Communications, vol. 8, no. 8, pp. 927-934, 2003.

[6] A. Hassinen, I. Moreels, K. de Nolf, P. F. Smet, J. C. Martins, and Z. Hens, "Short-chain alcohols strip X-type ligands and quench the luminescence of PbSe and CdSe quantum dots, acetonitrile does not," Journal of the American Chemical Society, vol. 134, no. 51, pp. 20705-20712, 2012.

[7] D. Li, H. Yun, B. T. Diroll, V. V. T. Doan-Nguyen, J. M. Kikkawa, and C. B. Murray, "Synthesis and size-selective precipitation of monodisperse nonstoichiometric $\mathrm{M}_{\mathrm{x}} \mathrm{Fe}_{3-\mathrm{x}} \mathrm{O}_{4}$ $(\mathrm{M}=\mathrm{Mn}, \mathrm{Co})$ nanocrystals and their $\mathrm{DC}$ and $\mathrm{AC}$ magnetic properties," Chemistry of Materials, vol. 28, no. 2, pp. 480 489, 2016.

[8] W. Zhao, L. Lin, and I.-M. Hsing, "Nucleotide-mediated size fractionation of gold nanoparticles in aqueous solutions," Langmuir, vol. 26, no. 10, pp. 7405-7409, 2010.

[9] L. Wu, J. Zhang, and W. Watanabe, "Physical and chemical stability of drug nanoparticles," Advanced Drug Delivery Reviews, vol. 63, no. 6, pp. 456-469, 2011.

[10] L. Patil and P. R. Gogate, "Ultrasound assisted synthesis of stable oil in milk emulsion: study of operating parameters and scale-up aspects," Ultrasonics Sonochemistry, vol. 40, Part A, pp. 135-146, 2018.

[11] D. Ramimoghadam, S. Bagheri, and S. B. Abd Hamid, "Stable monodisperse nanomagnetic colloidal suspensions: an overview," Colloids and Surfaces. B, Biointerfaces, vol. 133, pp. 388-411, 2015.

[12] P. Muneesawang and C. Sirisathitkul, "Size measurement of nanoparticle assembly using multilevel segmented TEM images," Journal of Nanomaterials, vol. 2015, Article ID 790508, 8 pages, 2015.

[13] S. Sun, "Recent advances in chemical synthesis self-assembly and applications of FePt nanoparticles," Advanced Materials, vol. 18, no. 4, pp. 393-403, 2006.

[14] A. Dong, X. Ye, J. Chen et al., "A generalized ligand-exchange strategy enabling sequential surface functionalization of colloidal nanocrystals," Journal of the American Chemical Society, vol. 133, no. 4, pp. 998-1006, 2011.

[15] Y. Shi, M. Lin, X. Jiang, and S. Liang, "Recent advances in FePt nanoparticles for biomedicine," Journal of Nanomaterials, vol. 2015, Article ID 467873, 13 pages, 2015.

[16] Y. Mori, "Size-selective separation techniques for nanoparticles in liquid," Kona Powder and Particle Journal, vol. 32, pp. 102-114, 2015. 


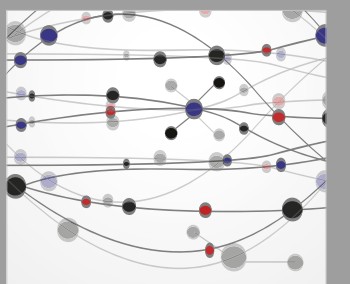

The Scientific World Journal
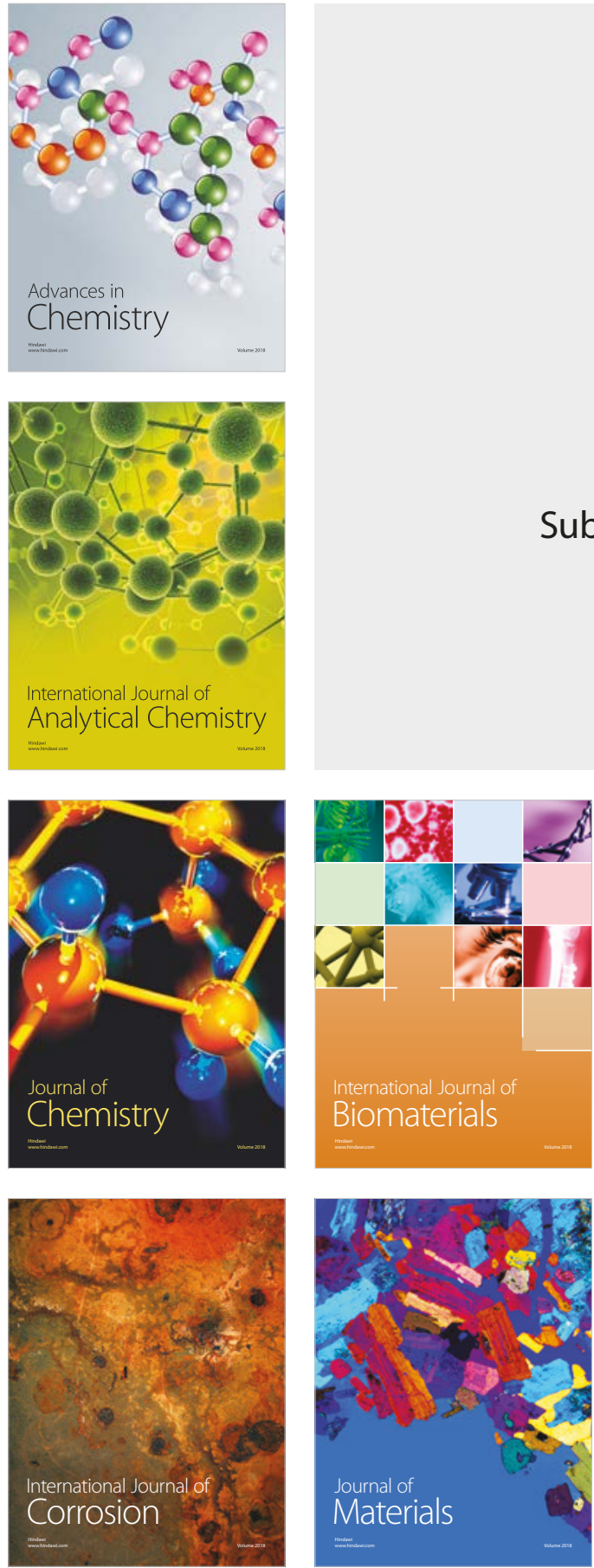

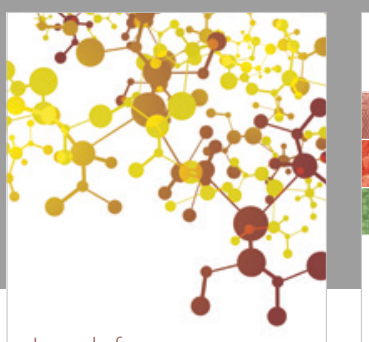

Journal of

Applied Chemistry
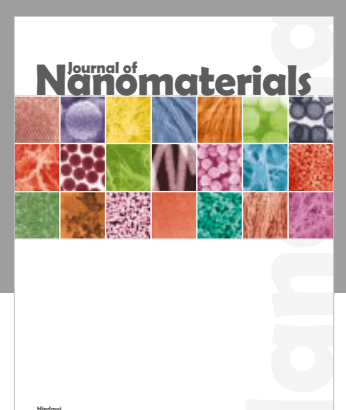

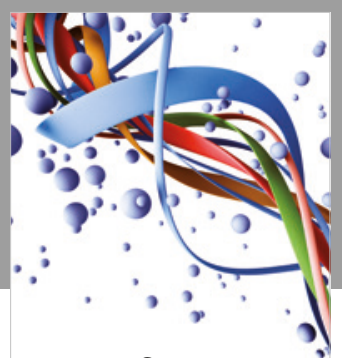

Scientifica

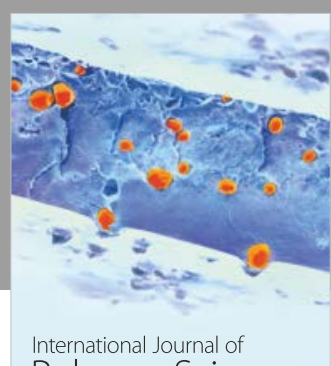

Polymer Science

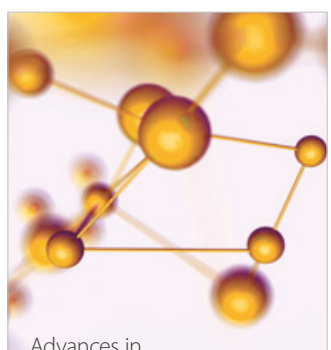

Physical Chemistry
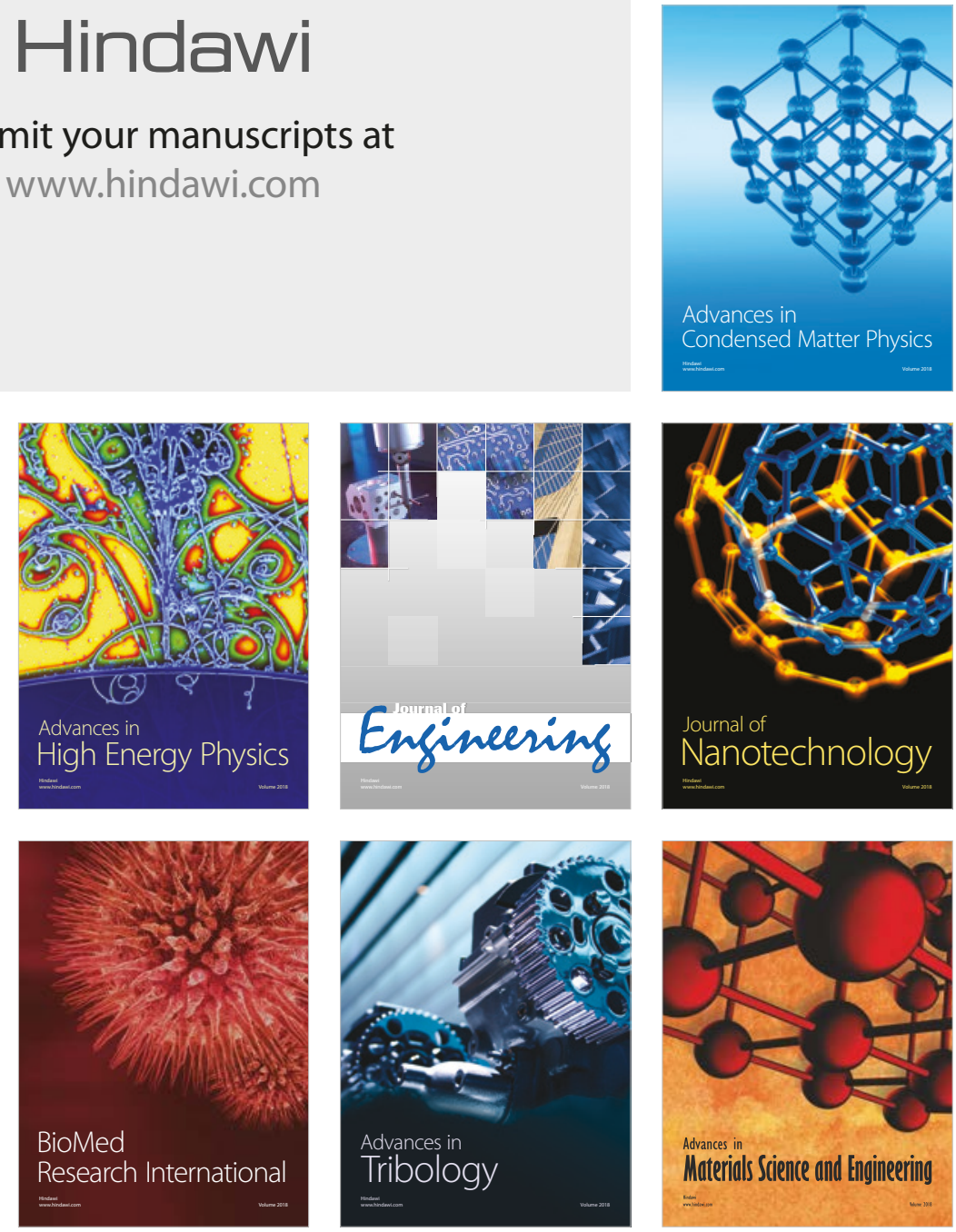